\title{
Compensation of transmission channel effects in chaos synchronization
}

\author{
A. Buscarino, L. Fortuna, M. Frasca, G. Sciuto \\ Dipartimento di Ingegneria Elettrica Elettronica e dei Sistemi, \\ University of Catania, Italy \\ and Laboratorio sui Sistemi Complessi, Scuola Superiore di Catania, \\ University of Catania, Italy.
}

\author{
M. T. Rashid \\ Electrical Engineering Department, \\ University of Basrah, \\ Basrah, Iraq.
}

\begin{abstract}
The synchronization of chaos is a well-known topic which attracted the attention of the scientific community in the last two decades. However, the robustness of the synchronous state has been not widely studied, especially considering real cases in which the effects introduced by the physical channel through which chaotic circuits interact, may deeply influence the quality of synchronization and even the onset of it. In this paper, the synchronization of two chaotic circuit coupled through a nonideal channel is investigated. In particular, the effects of channels introducing a frequency-independent or frequency-dependent time-delay are investigated. Furthermore, two different design strategies to obtain a linear compensation block able to compensate the considered channel effects are presented and the recovery of the synchronous state is discussed.
\end{abstract}

\section{INTRODUCTION}

Synchronization of two or more dynamical, in particular chaotic, systems is a phenomenon of great scientific interest in many different fields [1]. In particular, concerning synchronization of chaotic circuits, the first evidence has been observed and discussed in suitably coupled electronic circuits in [2]. Since then, different coupling schemes and techniques for the synchronization of nonlinear chaotic circuits have been developed.

Synchronization of chaos is based on the main idea that the suitable exchange of information between two or more circuits allows them to behave following a common dynamics [1]. However, the transmission channel along which coupled circuits interact introduces effects which may play a crucial role on the onset of synchronization.

In real cases, the transmission of the signal which couples two or more chaotic circuits has to be realized through a physical connection which may introduce distortion or undesired noise. The robustness of chaotic synchronization in presence of noisy transmission channel has been investigated in [3], where the suitability of chaos-based communication for binary signals is assessed. The effects introduced by a transmission channel in the synchronization of chaotic systems have been also studied in [4], [5] where the case of amplitude distortion and offset introduction are considered and a suitable recovery procedure presented.

Aim of this paper is to investigate the case in which the transmission channel introduces a different kind of distortion, i.e. the introduction of a time-delay. Moreover, the

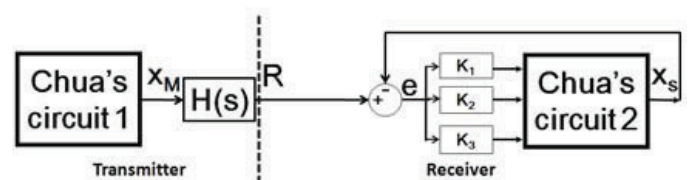

Fig. 1. Synchronization scheme of two Chua's circuit in presence of a nonideal transmission channel $H(s)$.

synchronization of two Chua's circuits [6], coupled through a Master-Slave configuration, is considered when the channel provides either a frequency-independent or a frequencydependent time-delay. The effects of the introduction of a distortion on the coupling signal will be characterized in terms of suitably defined synchronization indices. Two strategies for the compensation of the two considered effects will be outlined, showing the possibility to recover the synchronous state even in presence of non-ideal transmission channel.

The paper is organized as follows: in Section II the adopted synchronization scheme is described; in Section III the effect of a frequency-independent time-delay on synchronization is investigated and the compensation strategy discussed; in Section IV the effect of a frequency-dependent time-delay on synchronization is investigated and the compensation strategy discussed; finally, Section V draws some concluding remarks.

\section{SYNCHRONIZATION SCHEME}

The analysis of time-delay on the synchronization between two chaotic systems has been performed considering as case study a paradigmatic example of dynamical systems which may show chaotic behavior, i.e the Chua's circuit [6]. It represents the first example of electronic circuit in which chaos can be observed.

The synchronization scheme adopted to couple the two considered Chua's circuits is reported in Fig. 1. It is based on a Master-Slave configuration, hence, one Chua's circuit acts as the Master driving the other, i.e. the Slave, through a specific scalar signal. In particular, a negative feedback scheme has been adopted. In this case, the driving signal is used to create an error which is fed back in the slave system.

Particular attention should be given to properly choose the driving signal, since the onset of synchronization is strictly 
related to this choice. Many strategies have been developed to select the suitable coupling parameters in order to observe a synchronous behavior [1]. Among these, the Master Stability Function (MSF) approach [7] provides a simple method to select the coupling parameters. Starting from the MSF approach, the two Chua's circuits have been coupled by using as driving signal the first state variable of the master system and by building an error signal comparing the correspondent variable of the slave system [8]. The obtained scalar error signal has been fed back to the three state equation of the slave. Hence, the two coupled circuits can be described by the following equations:

$$
\left\{\begin{array}{l}
\dot{x}_{M}(t)=\alpha\left[y_{M}(t)-x_{M}(t)-h\left(x_{M}(t)\right)\right] \\
\dot{y}_{M}(t)=x_{M}(t)-y_{M}(t)+z_{M}(t) \\
\dot{z}_{M}(t)=-\beta y_{M}(t)-\gamma z_{M}(t) \\
\dot{x}_{S}(t)=\alpha\left[y_{S}(t)-x_{S}(t)-h\left(x_{S}(t)\right)\right]+k\left(R(t)-x_{S}(t)\right) \\
\dot{y}_{S}(t)=x_{S}(t)-y_{S}(t)+z_{S}(t)+k\left(R(t)-x_{S}(t)\right) \\
\dot{z}_{S}(t)=-\beta y_{S}(t)-\gamma z_{S}(t)+k\left(R(t)-x_{S}(t)\right)
\end{array}\right.
$$

where $h(x)=m_{1} x+0.5\left(m_{0}-m_{1}\right)(|x+1|-|x-1|)$ is the systems nonlinearity, $\alpha=9, \beta=14.286, \gamma=0$, $m_{0}=-1 / 7$, and $m_{1}=2 / 7$ are system parameters, $k$ is the coupling strength and $R(t)$ is the driving signal as received by the slave. In absence of coupling, the two circuits show a chaotic behavior.

Let $H(s)$ be the transfer function of the transmission channel. If the channel does not introduce any effect on the transmitted signal, i.e. in the ideal case, we can consider $H(s)=1$, and therefore $R(t)=x_{M}(t)$. In this case, fixing the coupling strength $k=5$ complete synchronization can be observed.

However, in real cases, $H(s)$ may introduce amplitude and phase distortions which can affect the onset of complete synchronization. In the following, the synchronization of the two Chua's circuits will be characterized with respect to two possible transmission channel transfer functions, both introducing a time-delay.

Let $S(t)=x_{M}(t)$ be the driving signal sent by the master. It will pass trough the transmission channel and will be received by the slave as $R(t)$.

Two possible effects are now taken into account: the introduction of a frequency-independent time-delay, and the presence of a frequency-dependent phase distortion due to the channel transfer function. Moreover, as shown in Fig. 2, aim of this paper is also to propose a design strategy for suitable linear dynamical systems able to recover the correct information in order to observe a synchronous state, compensating the effect of the transmission channel.

\section{COMPENSATION OF A FREQUENCY-INDEPENDENT TIME-DELAY}

As a first case study, let us consider the introduction of a frequency-independent time-delay $\tau$, i.e. the transmission channel can be considered as an ideal time-delay $H(s)=$ $e^{-s \tau}$. In this case the received signal is $R(t)=S(t-\tau)=$

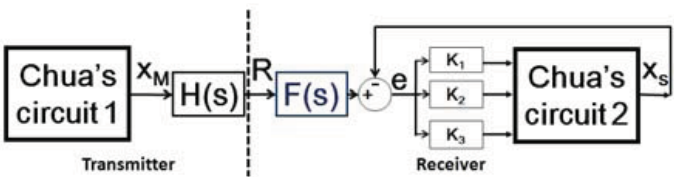

Fig. 2. Synchronization scheme of two Chua's circuit in presence of a nonideal transmission channel $H(s)$ and of the compensation block $F(s)$.

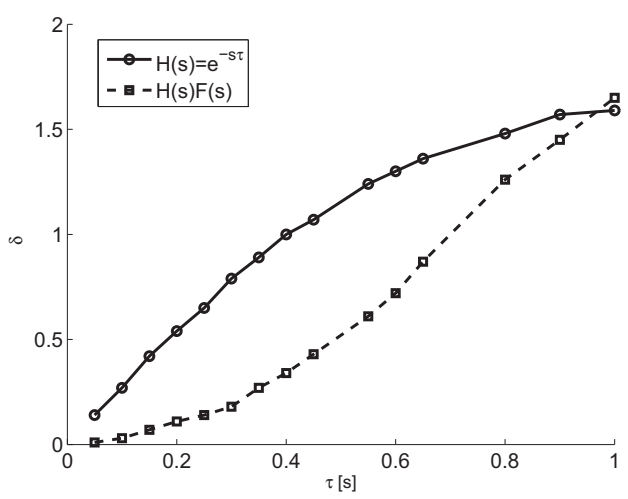

Fig. 3. Synchronization of two Chua's circuit in presence of frequencyindependent time-delay introduced by the transmission channel: synchronization error $\langle\delta\rangle$ as a function of the time-delay $\tau$ without (continuous line) and with (dotted line) compensation block $F(s)$.

$x_{M}(t-\tau)$ and, in general, complete synchronization cannot be ensured.

The behavior of the two coupled circuits has been investigated with respect to increasing time-delay provided by the channel. Let us define the synchronization error as $\langle\delta\rangle=\left\langle\sqrt{\frac{\left|x_{M}-x_{S}\right|^{2}+\left|y_{M}-y_{S}\right|^{2}+\left|z_{M}-z_{S}\right|^{2}}{3}}\right\rangle$ where the operator $\langle\cdot\rangle$ identifies the average in time. The continuous line in Fig. 3 shows the trend of the synchronization error $\langle\delta\rangle$ as a function of the introduced time-delay $\tau$. A drastic increase of the synchronization error can be noticed even for small values of $\tau$.

Furthermore, it is interesting to observe that, even if complete synchronization cannot be observed, the chaotic behavior is preserved, as shown in Fig. 4, where the trend of the state variables $x$ of both master and slave systems are reported for the different values of $\tau$ indicated in the figure caption. Moreover, lag synchronization can be observed, hence the slave system can be considered as synchronized with a timedelayed copy of the master.

In order to compensate frequency-independent time-delays, a linear block with transfer function $F(s)$ based on the inverse of a Bessel filter has been designed. Bessel filters are linear active filters able to approximate an ideal delay in a given range of frequencies [9]. A second order Bessel filter is characterized by the following transfer function:

$$
B(s)=\frac{1}{c_{0}+c_{1} s+c_{2} s^{2}}
$$




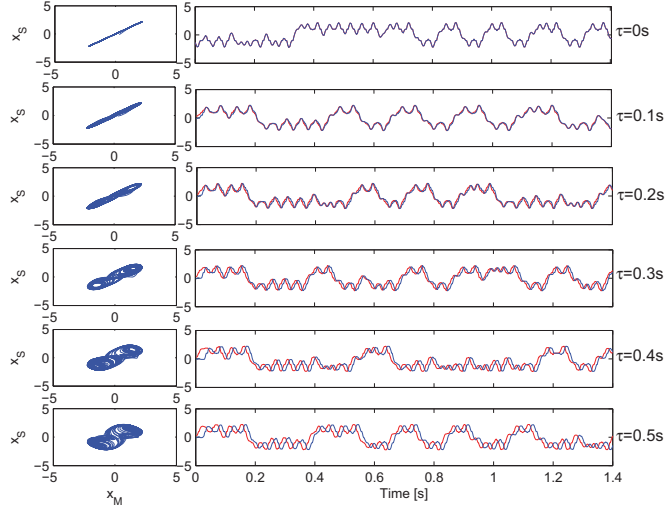

Fig. 4. Synchronization of two Chua's circuit in presence of time-delay introduced by the transmission channel: behavior of state variables $x_{M}$ and $x_{S}$ for different values of $\tau$, i.e. $\tau=0, \tau=0.1 s, \tau=0.2 s, \tau=0.3 s$, $\tau=0.4 s, \tau=0.5 s$

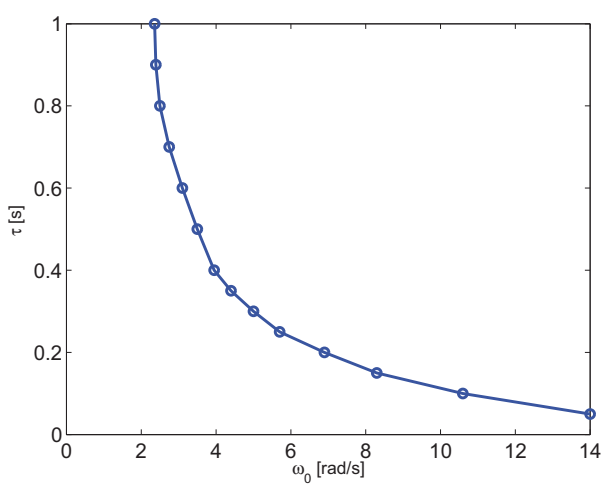

Fig. 5. Dependence of the $3 d B$ frequency $\omega_{0}$ of the Bessel filter on the actual time-delay implented.

where $c_{0}, c_{1}$, and $c_{2}$ are calculated as a function of the $3 d B$ frequency $\omega_{0}$. The main idea is that the inverse of $B(s)$ is able to compensate the phase distortion introduced by $H(s)$ up to $\omega_{0}$. Obviously, in order to obtain a realizable transfer function $F(s)$, three high frequency poles located at $p$ have to be added. Hence the complete transfer function of the compensation block is $F(s)=\frac{1}{B(s)} \frac{1}{\left(\frac{s}{p}+1\right)^{3}}$, where $p$ is higher enough to avoid effects on Chua's circuit dynamics, i.e. $p=40$.

Varying $\omega_{0}$ the corresponding second order Bessel filter is able to compensate a given delay as reported in Fig. 5 in which the time-delay $\tau$ is calculated as $\tau=-\frac{d \Phi(B(j \omega))}{d \omega}$. Thus, in order to compensate a time-delay $\tau=0.1 \mathrm{~s}$, the compensation block can be designed choosing $\omega_{0}=10.6 \mathrm{rad} / \mathrm{s}$ for which the coefficients of the corresponding Bessel filter are $c_{0}=1$, $c_{1}=0.163$, and $c_{2}=0.0089$. The Bode diagrams reported in Fig. 6 show that the compensation block is able to compensate the time-delay introduced by the transmission channel.

This strategy is effective to compensate time-delays up to $1 s$ as shown by the dotted line in Fig. 3 corresponding to the error index $\langle\delta\rangle$ in presence of the compensation block

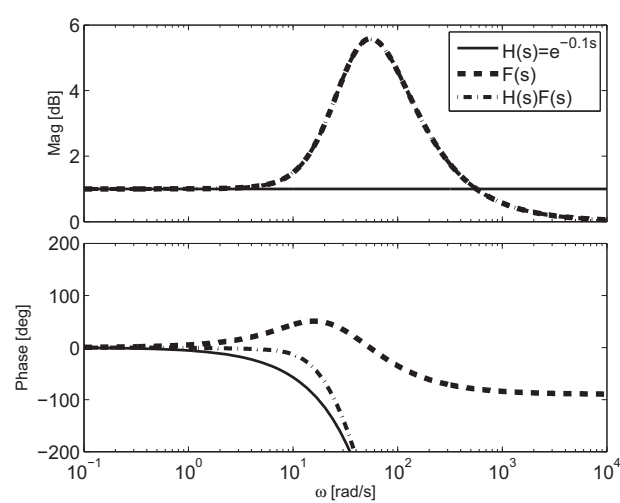

Fig. 6. Bode diagrams of the transmission channel $H(s)$ with $\tau=0.1 s$, the Bessel filter based compensator $F(s)$ for $\omega_{0}=10.6 \mathrm{rad} / \mathrm{s}$, and the resulting cascaded transfer function $C(s)$

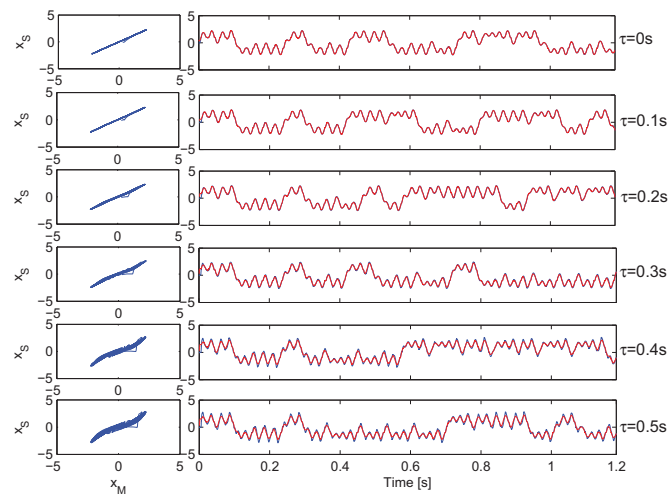

Fig. 7. Synchronization of two Chua's circuit in presence of time-delay introduced by the transmission channel with compensation block: behavior of state variables $x_{M}$ and $x_{S}$ for different values of $\tau$, i.e. $\tau=0, \tau=0.1 s$, $\tau=0.2 s, \tau=0.3 s, \tau=0.4 s, \tau=0.5 s$

designed according to Fig. 5. In Fig. 7 the trend of the $x_{M}$ and $x_{S}$ are reported for different values of $\tau$ showing that complete synchronization can be recovered even in presence of non-zero time-delays.

\section{COMPENSATION OF A FREQUENCY-DEPENDENT DISTORTION}

The second case considered takes into account that the transmission channel may introduce a frequency-dependent phase distortion. This effect can be studied approximating the transmission channel as an all-pass filter characterized by the following transfer function:

$$
H(s)=\frac{(a-s)}{(a+s)}
$$

where $a$ is the absolute value of the zero-pole pair location corresponding to the frequency at which input and output are in quadrature. 


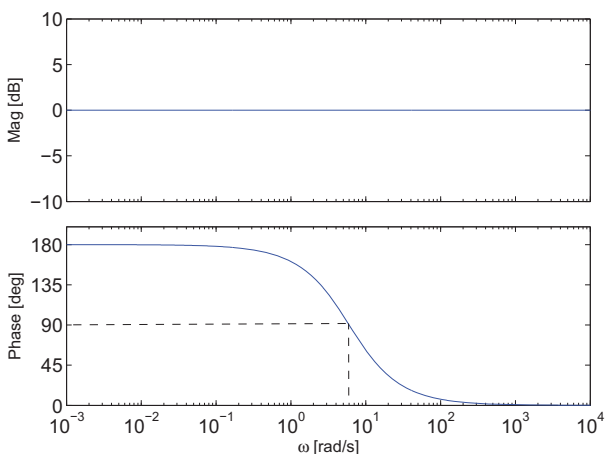

Fig. 8. Bode diagrams of an all-pass filter with transfer function $H(s)$ and $a=6$.

All-pass filters have a frequency response characterized by a flat unitary magnitude, i.e. amplitudes at all frequency remain unaltered, and a frequency-dependent phase shift, i.e. a propagation time-delay varying with the frequency, as shown by the Bode diagrams reported in Fig. 8, in which the frequency response of an all-pass filter with $a=6$ is shown. In particular, frequencies below (above) a decade from the zero-pole pair location are shifted by $\pi \operatorname{rad}(0 \mathrm{rad})$.

This behavior significantly affects the synchronization of the two coupled Chua's circuits, since it gives rise to antisynchronous behavior. In order to quantify the synchronization in this case, a peculiar error index, which tends to zero in both cases of complete and anti-synchronization, should be defined. Let us consider as synchronization error $\left\langle\delta_{a}\right\rangle=\left\langle\sqrt{\frac{\| x_{M}|-| x_{S}||^{2}+|| y_{M}|-| y_{S}||^{2}+|| z_{M}|-| z_{S}||^{2}}{3}}\right\rangle$ whose behavior with respect to different values of $a$ is reported in Fig. 9. It is interesting to note that when the all-pass filter has a quadrature frequency below the frequency range of the Chua's circuit, synchronization is preserved, otherwise anti-synchronization occurs. The main distorting effects are observed when the all-pass filter has a pole in the range of frequency of the Chua's circuit dynamics.

In order to compensate the all-pass-like effect which introduces a frequency-dependent phase distortion, the following compensating transfer function is introduced:

$$
F(s)=\frac{(a+s)^{2}}{(b+s)^{2}}
$$

where $a$ is the all-pass zero-pole pair location and $b$ is the location of two high frequency poles. The introduction of $F(s)$ allows to compensate the all-pass filter. In fact, cascading the two transfer function $H(s)$ and $F(s)$, one obtains:

$$
C(s)=H(s) F(s)=\frac{(a-s)}{(a+s)} \frac{(a+s)^{2}}{(b+s)^{2}}=\frac{(a-s)(a+s)}{(b+s)^{2}}
$$

As shown by the Bode diagrams reported in Fig. 10, the phase distortion observed considering the frequency response

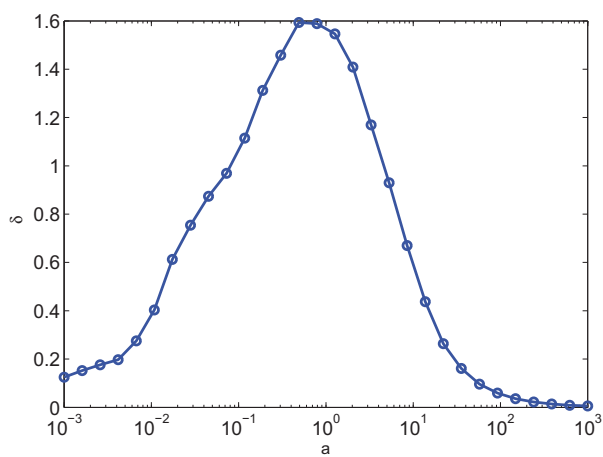

Fig. 9. Synchronization of two Chua's circuit in presence of frequencydependent time-delay introduced by the transmission channel: synchronization error $\left\langle\delta_{a}\right\rangle$ as a function of $a$.

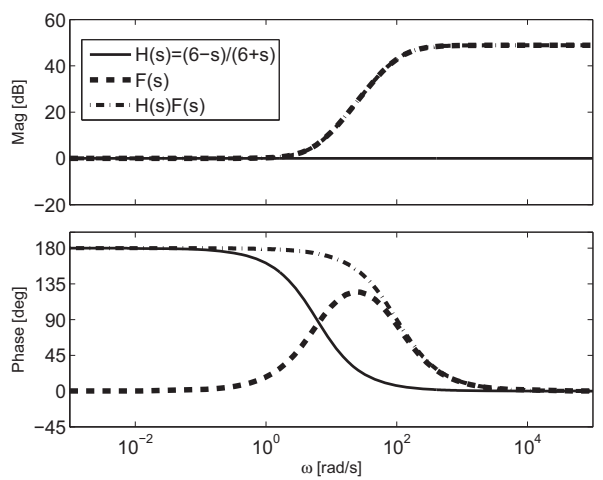

Fig. 10. Bode diagrams of the transmission channel $\mathrm{H}(\mathrm{s})$ with $a=6$, the compensation block $\mathrm{F}(\mathrm{s})$ in Eq. (4) with $a=6$ and $b=40$ and the resulting cascaded transfer function $C(s)$.

of $C(s)$ is shifted to higher frequency and, thus, outside the range of the Chua's circuit dynamics.

This approach reveals its effectiveness when $a \geq 2$. The results obtained with this strategy are summarized in Fig. 11 where the synchronization error $\left\langle\delta_{a}\right\rangle$ is reported without (continuous line) and with (dotted line) compensation.

\section{CONCLUSION}

In this paper, the role played by non-ideal transmission channel on the onset of synchronization of chaotic systems has been investigated. In particular, both frequency-independent and frequency-dependent time-delays introduced by the channel have been considered.

In the case of frequency-independent time-delays, the complete synchronization of the two circuit cannot be observed, even if a weaker form of synchronization, i.e. lag synchronization, occurs. A strategy based on Bessel filters has been outlined in order to design a compensation block able to recover the synchronous state even in presence of time-delay of $1 s$. 


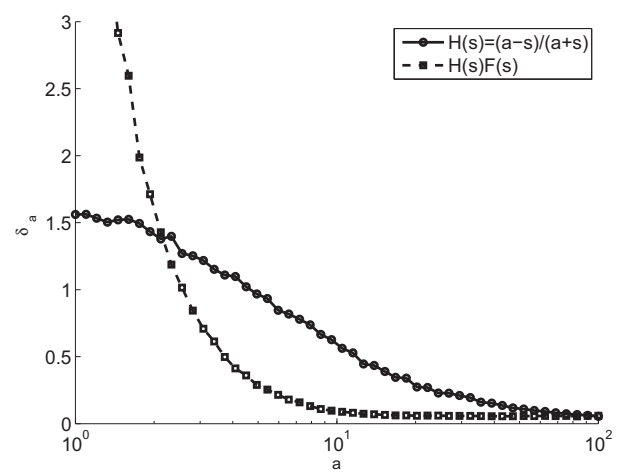

Fig. 11. Synchronization of two Chua's circuit in presence of frequencydependent time-delay introduced by the transmission channel: synchronization error $\left\langle\delta_{a}\right\rangle$ as a function of $a$ without (continuous line) and with (dotted line) compensation block $F(s)$.

As concerns frequency-dependent time-delay, the channel has been modeled using an all-pass transfer function which introduces a phase distortion depending on the signal frequency. In this case, the onset of an anti-synchronous behavior has been observed. When the channel introduces a time-delay for which synchronization is lost, the suitable strategy introduced allows to recover chaos synchronization.

\section{REFERENCES}

[1] A. Pikovsky, M. Rosenblum, J. Kurths, Synchronization: A Universal Concept in Nonlinear Sciences, Cambridge University Press, 2003.

[2] L. M. Pecora and T. L. Carroll, Phys. Rev. Lett. 64, 8211990.

[3] A. N. Miliou, I. P. Antoniades, S. G. Stavrinides, A. N. Anagnostopoulos, Secure communication by chaotic synchronization: Robustness under noisy conditions, Nonlinear Analysis: Real World Applications, 8, pp. 1003-1012, 2007

[4] T. X. Wu, D. L. Jaggard, Chaotic Wave Scattering and Synchronization, Proc. of Int. Symp. of Antennas and Propagation Society, 2, pp. 13881397,1999

[5] X. Yang, T. X. Wu, D. L. Jaggard, Synchronization Recovery of Chaotic Wave Through an Imperfect Channel, IEEE Antennas and Wireless Propagation Letters, 1, pp. 154-156, 2002.

[6] L. Fortuna, M. Frasca and M.G. Xibilia, Chua's Circuit Implementations: Yesterday, Today, Tomorrow, World Scientific, 2009.

[7] L. M. Pecora, T. L. Carroll, Master Stability Functions for Synchronized Coupled Systems, Phys. Rev. Lett., 80, pp. 2109-2112, 1998.

[8] A. Buscarino, L. Fortuna, M. Frasca, Chua's Circuits synchronization with diffusive coupling: new results, Int. J Bif. and Chaos, 19, pp. 3103-3107, 2009.

[9] G. Daryanani, Principles of active network synthesis and design, John Wiley \& sons, Singapore 1976. 